ARTÍCULO ORIGINAL

\title{
Frecuencia de anomalías congénitas en el Instituto Materno Infantil de Bogotá
}

\author{
Herbert García ${ }^{1}$, Gustavo Andrés Salguero ${ }^{1}$, Jeffer Moreno ${ }^{1}$, \\ Clara Arteaga ${ }^{2}$, Alejandro Giraldo ${ }^{3}$ \\ ${ }^{1}$ Instituto de Genética, Universidad Nacional de Colombia, Bogotá, D.C., Colombia. \\ ${ }^{2}$ Unidad de Biología de la Reproducción, Instituto Materno Infantil; Departamento de Ginecoobstetricia, \\ Facultad de Medicina, Universidad Nacional de Colombia, Bogotá, D.C., Colombia. \\ ${ }^{3}$ Departamento de Medicina Interna, Facultad de Medicina; Instituto de Genética, Universidad Nacional de \\ Colombia; Fundación Gillow, Bogotá, D.C., Colombia.
}

Se analizaron 5.686 nacimientos (5.597 vivos y 89 muertos) correspondientes a dos series realizadas entre los meses de octubre de 1997 y abril de 1998 y de julio a noviembre de 2000 (12 meses) en el Instituto Materno Infantil de Bogotá. Se detectaron anomalías congénitas en $4,4 \%$ de los recién nacidos vivos y en $7,8 \%$ de los mortinatos. Las anomalías mayores constituyen el $69 \%$ de todas las anomalías y se encuentran en $3 \%$ de los recién nacidos vivos. Las anomalías moderadas son el $31 \%$ de todas las anomalías y se detectaron en 1,4\% de los recién nacidos vivos. Los recién nacidos con anomalías mayores, en comparación con los controles, registran una mayor mortalidad al momento del alta hospitalaria $(p=0,0001)$, menor peso promedio al nacer $(p=0,003)$ y antecedentes de anomalía en la familia $(p=0,0001)$. Las anomalías moderadas están asociadas únicamente con antecedentes de anomalías familiares $(p=0,0001)$. Las anomalías congénitas muestran una frecuencia en nuestro medio semejante a las de otros estudios del país y del exterior, aunque, individualmente, algunas anomalías muestran variaciones significativas en sus frecuencias que pueden deberse a sobre 0 subregistros o a diferencias metodológicas en su detección.

Palabras clave: anomalías congénitas, malformaciones, deformaciones, defectos congénitos, vigilancia epidemiológica, monitorización.

\section{Frequency of congenital anomalies at the Instituto Materno Infantil, Bogotá, Colombia}

At the Instituto Materno Infantil (IMI) in Bogotá (Colombia), 5,686 births (5,597 live births and 89 stillbirths) were analyzed during two periods: from October, 1997, to April, 1998, and from July to November, 2000 (12 months). Congenital anomalies were detected in $4.4 \%$ of live newborn babies and in $7.8 \%$ of stillbirths. Major anomalies corresponded to $69 \%$ and mild anomalies to $31 \%$ (3\% and $1.4 \%$ of all live births, respectively). The newborn babies with major anomalies, in comparison to the normal controls, had higher mortality at hospital discharge $(p=0.0001)$, lower average birth weight $(p=0.003)$, and family history of congenital anomalies $(p=0.0001)$. The only significant association for mild anomalies was with family history of congenital anomalies $(p=0.0001)$. The frequency of congenital anomalies was similar to that in other studies, although certain kinds of anomalies showed noticeable frequency differences. This may bea consequence of differences in record keeping or in detection methods.

Key words: congenital anomalies, malformations, deformations, birth defects, epidemiological surveillance, monitoring.

\section{Correspondencia:}

Alejandro Giraldo, Instituto de Genética, oficina 214. Universidad Nacional, entrada calle 53, Bogotá, Colombia. Teléfono 316 5000, extensión 11610; fax 3165526. algirald@bacata.usc.unal.edu.co

Recibido: 03/12/02; aceptado: 04/05/03
La tasa de mortalidad infantil (TMI) en Colombia se ha estimado según diferentes metodologías. Para 1994, según las cifras de mortalidad del Dane y el censo de 1993, se calculó la TMI en 34,15\%, cifra que mostró un importante descenso (23\%) con respecto a 1985 (44,24\%) (1). Por otra parte, 
por medio de encuestas realizadas con muestras probabilísticas en mujeres en edad fértil, se ha estimado la TMI en $44 \%$ para el quinquenio 1975 1980 y en $21 \%$ para el quinquenio $1995-2000$, lo que ha mostrado una disminución de más del $50 \%$ en esos 25 años (2). Las anteriores cifras, aunque discrepantes, muestran una definida tendencia a la baja. Esta disminución en las TMI genera cambios importantes en las causas. Las anomalías congénitas están entre las cinco primeras causas de mortalidad en menores de un año en varios países en desarrollo. En los países desarrollados son la primera o segunda causa de mortalidad infantil (3). En Estados Unidos, la mortalidad por anomalías congénitas ha aumentado de $7 \%$ en 1916 a $21 \%$ en 1988 . Desde entonces, es la primera causa de mortalidad infantil, seguida de las condiciones relacionadas con la prematurez y el síndrome de muerte súbita (4).

La evidencia disponible señala que la prevalencia y la carga potencial de los desórdenes congénitos en la salud pública de los países en desarrollo es elevada y en algunas instancias mayor que en los países desarrollados. Los países latinoamericanos, en los que la mortalidad infantil ha caído por debajo de 50/1.000, las anomalías congénitas ocupan el tercer puesto y han adquirido significación como problema de salud pública.

Las anomalías congénitas y los trastornos hereditarios son los responsables de $19 \%$ de las muertes en las unidades de cuidados intensivos pediátricos (5). Así mismo, se ha observado que entre las causas de muertes posneonatales (28364 días) de niños de más de $500 \mathrm{~g}$ de peso al nacimiento, las condiciones genéticas ocupan el segundo lugar ( $20 \%)$, después de la muerte súbita $(47 \%)$ y seguidas por las condiciones relacionadas con prematurez (11\%) e infecciones (9\%) (6).

Se ha estimado que el costo del tratamiento y manejo de las anomalías congénitas en Estados Unidos sobrepasa anualmente los US\$6 mil millones, sin incluir costos no médicos que cubren las familias (7).

Es de igual importancia en nuestro medio considerar la carga familiar que representa un niño con anomalías congénitas, no sólo desde el punto de vista médico sino psicológico. Se crea estrés familiar por miedo a la estigmatización, a la falta de cobertura de las EPS y, en algunos casos, a la dificultad para entender adecuadamente la información acerca de la anomalía del hijo afectado, lo cual contribuye al manejo inapropiado de estos niños.

Al analizar 56 causas de mortalidad infantil en Colombia, las anomalías congénitas que se encontraban en el séptimo lugar en la década de 1970 suben al cuarto lugar en la década de 1980 y al tercer lugar en 1994 (1). Esto se hace más evidente por la disminución de la mortalidad debida a causas infecciosas y el empleo de mejores métodos de diagnóstico. Por otra parte, el indicador de carga de la enfermedad 'años de vida saludables perdidos' (AVISA) muestra, para toda la población colombiana y para todas las edades, un quinto lugar para las anomalías congénitas, después de homicidio y violencia, afecciones perinatales, accidentes de tránsito y enfermedad isquémica cardiaca, que ocupan los primeros cuatro lugares (8). Lo anterior indica la particular importancia de conocer la frecuencia y los factores de riesgo asociados con la ocurrencia de las anomalías congénitas en el país y en las principales ciudades. En Bogotá, las anomalías congénitas son la segunda causa de mortalidad infantil desde mediados de la década de 1980 y la primera causa de AVISA en menores de cinco años (9).

Los sistemas de vigilancia epidemiológica continúan siendo muy eficaces para conocer aspectos médicos, genéticos y epidemiológicos de las anomalías congénitas y han permitido diseñar programas de prevención y de rehabilitación (10).

En la mayoría de los países europeos y en particular los pioneros en establecer sistemas de vigilancia epidemiológica, la información sobre las anomalías congénitas es obligatoria. Ésta se obtiene a partir de las historias de los nacimientos, que son hospitalarios prácticamente en su totalidad, así como de otros registros médicos en diferentes edades (11-13).

A partir de 1974, los sistemas de vigilancia epidemiológica de varios países fueron agrupados 
por la International Clearinghouse for Birth Defects Monitoring Systems (ICBDMS) con el fin de homologar los diferentes métodos de clasificación y análisis de las anomalías congénitas, agilizar el intercambio de información y permitir su comparación $(14,15)$.

Posteriormente surge, además, el EUROCAT, agrupación de la Comunidad Económica Europea que cobija específicamente a los sistemas de vigilancia de ese continente $(16,17)$. La ICBDMS y el EUROCAT realizan actividades conjuntas y reuniones periódicas (18).

Por otra parte, el Centro de Control de Enfermedades de Atlanta inició en 1967 el Programa de Defectos Congénitos del Área Metropolitana de Atlanta (MACDP), el primero de dos programas independientes que buscan estudiar el problema de las anomalías congénitas en ese país.

En 1967 se inició el Estudio Colaborativo Latinoamericano de Malformaciones Congénitas (ECLAMC), el cual en diferentes épocas ha cubierto 12 países, incluido Colombia $(19,20)$, que evalúa aproximadamente el $1 \%$ de los nacimientos de cada país. En México, el Registro y Vigilancia Epidemiológica de Malformaciones Congénitas Externas (RYVEMCE) cubre aproximadamente el $2 \%$ de los nacimientos para ese país (21).

En Colombia, en 1992 se propuso el programa de Vigilancia Epidemiológica y Registro de Anomalías Congénitas de Colombia (VERACC) como iniciativa del Instituto Nacional de Salud, con base y con autorización del ECLAMC, el cual cubría varias ciudades, pero al cabo de dos años se suspendieron sus actividades. Actualmente se carece de un registro sistemático en nuestro país. No obstante, nuestro grupo consideró importante realizar en dos ocasiones (de octubre de 1997 a abril de 1998 y de julio a noviembre de 2000) el registro de series de nacimientos, para observar en esos dos períodos el comportamiento epidemiológico de las anomalías congénitas en el Instituto Materno Infantil de Bogotá, centro hospitalario de los Departamentos de Ginecobstetricia y Neonatología de la Facultad de Medicina de la Universidad Nacional de Colombia.

\section{Materiales y métodos}

EI VERACC al igual que el ECLAMC recoge información de nacimientos hospitalarios, cuyos datos se obtienen según un manual operacional en dos modalidades complementarias (19). Una de casos y controles que indaga múltiples variables y busca factores de riesgo y otra de monitorización o de cohorte, que averigua datos básicos y busca únicamente frecuencias.

Los datos familiares y los factores de riesgo se obtuvieron de médicos genetistas, pediatras 0 médicos internos y directamente de las madres de los niños, tanto en los casos como en los controles.

Se definieron los casos como los recién nacidos hospitalarios vivos o muertos mayores de $500 \mathrm{~g}$, con anomalías congénitas clínicamente detectables y los controles como los recién nacidos vivos mayores de $500 \mathrm{~g}$, del mismo sexo y sin anomalías congénitas, que nacieron inmediatamente después del niño con anomalías.

La descripción de esas anomalías y demás aspectos pertinentes se hizo según lo indicado en el manual operacional. Se tuvo especial atención en anotar todos los datos de los libros de partos, con el fin de obtener la información de base completa sobre todos los recién nacidos y así elaborar los informes mensuales de nacimientos, que constituyen el denominador de todas las variables del análisis.

Para la clasificación general de las anomalías se utilizó la Clasificación Internacional de Enfermedades (CIE-9) , modificada por la British Paediatric Association (22), la cual es prácticamente la misma que se utiliza actualmente en el registro de anomalías congénitas de Nueva York (23), basada en sistemas, pero que también incluye criterios anatómicos y etiológicos; éstos aseguran validez epidemiológica mundial y, además, categorización, aunque no se logre una descripción exacta. Se codificó hasta con dos dígitos adicionales a las categorías principales (cinco dígitos).

Se tuvieron en cuenta las definiciones Spranger (24) y Opitz $(25,26)$ para calificar todas las anomalías congénitas con sus diferentes 
modalidades: malformaciones, deformaciones, disrupciones y displasias, así como conjuntos de anomalías congénitas: secuencias, síndromes y asociaciones.

Adicionalmente, se definieron como anomalías mayores las alteraciones estructurales con un compromiso estético y funcional importante, que requieren un manejo médico-quirúrgico especializado, como la comunicación interventricular, el labio hendido y la displasia de cadera.

Las anomalías moderadas son aquellas malformaciones que indican cierta alteración en la formación de una estructura, se originan durante la organogénesis y se expresan como fenómenos de todo o nada. Pueden indicar o no alteraciones en otras estructuras asociadas, pero sin un compromiso funcional o estético importante; no necesitan manejo médico-quirúrgico o se corrigen fácilmente por cirugía estética, por ejemplo, los apéndices y fositas preauriculares, las polidactilias preaxiales pediculadas y las mamilas supernumerarias (26-28).

No se registraron en forma independiente en el estudio los niños con anomalías menores, las cuales son variables continuas del desarrollo y de la fenogénesis, que pueden ser de tipo étnico o de carácter familiar, tales como el epicanto, los pliegues transversos de las manos y las clinodactilias leves del quinto dedo, y que pueden presentarse en asociación con anomalías mayores o con patrones de anomalías (asociación, secuencia, síndromes) y son importantes como sugestivas de la presencia de estas alteraciones. Sin embargo, cuando se observan en forma aislada y sin asociación con anomalías mayores o moderadas no tienen significado clínico. Por esta razón, no se consideraron en el presente estudio $(24,27)$.

Aunque cualquier rasgo es el resultado de la acción combinada de factores genéticos y ambientales, se clasificaron los defectos congénitos según el catálogo de McKusick (Mendelian Inheritance in Man) (29), en las siguientes categorías: monogénicos (MG); multifactoriales o determinados por poligenes y factores ambientales (MF); esporádicos o de ocurrencia casual (ES); cromosómicos $(\mathrm{CR})$ y heterogéneos $(\mathrm{HT})$, así como aquéllos con evidencia de herencia monogénica, pero que pueden ser de origen esporádico o de origen ambiental o teratogénico.

Para la inclusión de algunas anomalías como la displasia congénita de la cadera, se consideraron sólo aquéllas en que la cadera fuera luxable (signo de Ortolani y de Barlow), se descartaron el click, y las asimetrías de pliegues. Se consideraron los apéndices preauriculares tanto sésiles como pediculados. El síndrome de Down se registró con el criterio clínico únicamente, aunque la mayoría de los casos fueron confirmados por cariotipo.

Se clasificaron como senos dérmicos pilonidales o sacrococcígeos aquéllos sin evidencia clínica de comunicación con el espacio subaracnoideo y sin otros hallazgos relacionados. Para el pie equino varo, se descartó que la deformación fuera posicional y que no redujera de manera activa.

El Grupo de Genética recibió la información mensualmente, revisándola para controlar errores e inconsistencias y archivándola en computadores por medio de base de datos en Access ${ }^{\circledR}$. Los análisis estadísticos se realizaron con el programa Stata ${ }^{\circledR} 6.0$.

Se analizaron las siguientes variables: peso, en las modalidades peso total, bajo peso $(<2.500 \mathrm{~g})$, muy bajo peso $(<1.500 \mathrm{~g})$; situación del paciente al ser dado de alta: vivo o muerto; sexo: masculino, femenino o ambiguo; presentación: cefálica, podálica u otra; parto: vaginal, cesárea, otro; antecedente de aborto; gravidez; edad materna; edad paterna; consanguinidad; antecedente de anomalías congénitas en la familia e ingestión de vitaminas periconcepcional. Con respecto al primer trimestre de la gestación, se registraron los antecedentes de enfermedades agudas (incluida la gripa), inmunizaciones durante el embarazo, enfermedades crónicas, exposición a factores físicos o químicos (uso de medicamentos, alcohol, drogas, cigarrillos y exposición a plaguicidas) y ocurrencia de metrorragia.

Para el análisis entre los casos y los controles, se utilizó el ji cuadrado para variables categóricas y la t de Student y Anova para variables continuas. 
Se utilizó un intervalo de confianza de 0,95 y se consideró valor $p<0,05$ para la significancia estadística.

Se utilizó distribución de Poisson para comparar las frecuencias halladas en un estudio previo realizado en el IMI en 1992 por Arteaga (30) y con las frecuencias observadas en el período 1982-1998 del ECLAMC (31). Se consideró un intervalo de confianza de $99 \%$ utilizando Stata 6.0 (32).

\section{Resultados}

La primera serie (octubre de 1997 a abril de 1998) estuvo compuesta por 3.921 nacimientos, con una media de 603,2 nacimientos por mes y un promedio de 20,1 nacimientos diarios. La segunda serie (julio a noviembre de 2000) fue de 1.765 nacimientos con un promedio de 352 nacimientos por mes y de 11,5 nacidos diarios. En la primera serie, la frecuencia de anomalías mayores fue de $118(3,05 \%)$ y la de las menores de $43(1,11 \%)$; en la segunda serie, las frecuencias fueron de 51 $(2,89)$ y $33(1,87 \%)$, respectivamente. Las dos series mostraron frecuencias de malformaciones totales cuyas diferencias no son significativas $(p=0,2986)$ y frecuencias de anomalías mayores que tampoco mostraron diferencias significativas $(p=0,7548)$. Dada la similitud, las dos series se unieron para realizar un análisis conjunto entre casos y controles. Se analizaron, entonces, un total de 5.686 nacimientos en los 12 meses antes mencionados.

El grupo de edad entre los 15 y 25 años tuvo el mayor número de nacimientos $(p<0,00001)$ y correspondió a las pacientes en su primera gestación ( $p<0,00001)$.

Del total de los 5.686 nacimientos, 89 fueron mortinatos; entre los 5.597 nacidos vivos, se encontraron 245 niños con anomalías (4,4\%). Por otra parte, 7 de los 89 mortinatos presentaron anomalías congénitas (7,8\%).

De todas las anomalías, las anomalías mayores correspondieron a $69 \%$ y a $3 \%$ de todos los nacimientos, mientras que las anomalías moderadas correspondieron a $31 \%$ de todas las anomalías y representaron $1,4 \%$ de todos los nacimientos.
Un $2 \%$ de las anomalías correspondieron a herencia de tipo mendeliana o monogénica, 30,2\% a herencia de tipo multifactorial o poligénica, $7,1 \%$ a alteraciones cromosómicas y $54,8 \%$ fueron heterogéneas. Un caso fue ocasionado probablemente por exposición materna comprobada a fenitoína (paciente con una anomalía de Ebstein).

En el grupo de las anomalías mayores se encontraron en orden descendente las siguientes frecuencias: displasia congénita de la cadera $(18,7 \%)$, síndrome de Down $(6,95 \%)$, pie equino varo $(6,25 \%)$, microtia $(3,40 \%)$, testículos no descendidos $(3,40 \%)$ e hidrocefalia $(2,84 \%)$ (cuadro 1). Entre las anomalías moderadas, los apéndices preauriculares ocupan el primer lugar con $27,63 \%$, seguidos por los senos coccígeos con $14,47 \%$, la polidactilia postaxial, $14,47 \%$, las fístulas preauriculares, $11,84 \%$ y los hemangiomas cutáneos, 5,36\% (cuadro 2).

Se halló una diferencia significativa entre la presencia de anomalías y el bajo peso al nacer (menor a $2.500 \mathrm{~g})(p=0,0001)$, aunque no se encontró relación con el muy bajo peso al nacer (menor a $1.500 \mathrm{~g})(p=0,823)$. Se detectó una diferencia significativa entre el promedio del peso de los malformados $(2.746,78 \mathrm{~g})$ y el promedio de peso de los controles $(2.875,33 \mathrm{~g})(p=0,0228)$. Se encontró asociación entre la condición de muerto al ser dado de alta (nativivos fallecidos) y la presencia de anomalías congénitas $(p=0,0001)$ y con el antecedente de anomalías en parientes de primer grado $(p=0,0001)$ (cuadro 3$)$. El análisis de las anomalías mayores mostró diferencias significativas en las variables mencionadas.

El análisis de las anomalías moderadas sólo mostró asociación significativa con el antecedente de anomalía familiar $(p=0,0001)$.

\section{Discusión}

Las dos series analizadas conjuntamente en el presente estudio (octubre 1997 a abril de 1998 y julio a noviembre de 2000), aunque representan dos grupos que difieren en el número de nacimientos diarios, fueron realizadas con los mismos criterios diagnósticos y de inclusión y no mostraron diferencias significativas en su 
Cuadro 1. Anomalías congénitas mayores, clasificadas de acuerdo con el Código Internacional de las Enfermedades 9 (CIE-9)

\begin{tabular}{|c|c|c|c|c|c|}
\hline CIE-9 & Descripción de la malformación & & $\begin{array}{l}\text { encia } \\
\quad \%\end{array}$ & $\begin{array}{c}\text { Tasa } \\
10.000\end{array}$ & $\begin{array}{l}\text { Nacimientos } x \\
\text { caso }\end{array}$ \\
\hline 238 & Neoplasia de origen incierto (teratoma) & 1 & 0,57 & 1,76 & $1: 5.686$ \\
\hline 740.02 & Anencefalia†† & 4 & 2,27 & 7,03 & $1: 1.422$ \\
\hline 741 & Espina bífida con hidrocefalia & 1 & 0,57 & 1,76 & $1: 5.686$ \\
\hline 741.01 & Espina bífida con malformación Arnold Chiari & 1 & 0,57 & 1,76 & $1: 5.686$ \\
\hline 741.04 & Espina bífida torácica con hidrocefalia & 2 & 1,14 & 3,52 & $1: 2.843$ \\
\hline 741.05 & Espina bífida lumbar con hidrocefalia† & 2 & 1,14 & 3,52 & $1: 2.843$ \\
\hline 741.93 & Espina bífida lumbar sin hidrocefalia & 2 & 1,14 & 3,52 & $1: 2.843$ \\
\hline 741.94 & Espina bífida sacra sin hidrocefalia & 1 & 0,57 & 1,76 & $1: 5.686$ \\
\hline 742.0 & Encefalocele occipital & 2 & 1,14 & 3,52 & $1: 2.843$ \\
\hline 742.1 & Microcefalia & 2 & 1,14 & 3,52 & $1: 2.843$ \\
\hline 742.26 & Holoprosencefalia & 3 & 1,70 & 5,28 & $1: 1.895$ \\
\hline 742.3 & Hidrocefalia congénita & 5 & 2,84 & 8,79 & $1: 1.137$ \\
\hline 742.31 & Síndrome Dandy Walker & 1 & 0,57 & 1,76 & $1: 5.686$ \\
\hline 742.39 & Hidrocefalia no específica & 3 & 1,70 & 5,28 & $1: 1.895$ \\
\hline 742.4 & Otras anomalías del cerebro & 1 & 0,57 & 1,76 & $1: 5.686$ \\
\hline 742.40 & Macrocefalia & 1 & 0,57 & 1,76 & $1: 5.686$ \\
\hline 744.21 & Microtia & 6 & 3,41 & 10,55 & $1: 948$ \\
\hline 745.3 & Ventrículo común & 1 & 0,57 & 1,76 & $1: 5.686$ \\
\hline 745.4 & Defecto septal ventricular & 3 & 1,70 & 5,28 & $1: 1.895$ \\
\hline 745.61 & Aurícula común/única & 1 & 0,57 & 1,76 & $1: 5.686$ \\
\hline 746.1 & Atresia tricuspídea congénita & 1 & 0,57 & 1,76 & $1: 5.686$ \\
\hline 746.2 & Anomalía de Ebstein & 1 & 0,57 & 1,76 & $1: 5.686$ \\
\hline 746.6 & Insuficiencia mitral congénita & 1 & 0,57 & 1,76 & $1: 5.686$ \\
\hline 746.7 & Síndrome de corazón izquierdo hipoplásico & 1 & 0,57 & 1,76 & $1: 5.686$ \\
\hline 746.87 & Bloqueo cardiaco congénito & 1 & 0,57 & 1,76 & $1: 5.686$ \\
\hline 747.5 & Arteria umbilical única & 1 & 0,57 & 1,76 & $1: 5.686$ \\
\hline 748.12 & Nariz fisurada & 1 & 0,57 & 1,76 & $1: 5.686$ \\
\hline 749.2 & Labio y paladar hendido & 4 & 2,27 & 7,03 & $1: 1.422$ \\
\hline 750.3 & Estenosis esofágica sin fístula T-E & 4 & 2,27 & 7,03 & $1: 1.422$ \\
\hline 750.31 & Estenosis esofágica con fístula T-E & 2 & 1,14 & 3,52 & $1: 2.843$ \\
\hline 751.1 & Estenosis del duodeno & 1 & 0,57 & 1,76 & $1: 5.686$ \\
\hline 751.11 & Estenosis del yeyuno & 1 & 0,57 & 1,76 & $1: 5.686$ \\
\hline 751.12 & Estenosis del íleon & 1 & 0,57 & 1,76 & $1: 5.686$ \\
\hline 751.54 & Fístula anal congénita & 1 & 0,57 & 1,76 & $1: 5.686$ \\
\hline 752.51 & Testículos no descendidos bilaterales & 6 & 3,41 & 10,55 & $1: 948$ \\
\hline 752.6 & Hipospadias & 1 & 0,57 & 1,76 & $1: 5.686$ \\
\hline 752.9 & $\begin{array}{l}\text { Otras anomalías no específicas de genitales } \\
\text { (masa escrotal) }\end{array}$ & 1 & 0,57 & 1,76 & $1: 5.686$ \\
\hline 753 & Secuencia de Potter† & 2 & 1,14 & 3,52 & $1: 2.843$ \\
\hline 753.01 & Ausencia, agenesia o displasia unilateral de riñón & 1 & 0,57 & 1,76 & $1: 5.686$ \\
\hline 753.11 & Riñón poliquístico & 2 & 1,14 & 3,52 & $1: 2.843$ \\
\hline 753.16 & Rinón multiquístico & 2 & 1,14 & 3,52 & $1: 2.843$ \\
\hline 753.2 & Hidronefrosis congénita & 2 & 1,14 & 3,52 & $1: 2.843$ \\
\hline 753.38 & Otras anomalías específicas de riñón (pelvis ectópica) & 1 & 0,57 & 1,76 & $1: 5.686$ \\
\hline 754.3 & Displasia congénita de la cadera & 33 & 18,75 & 58,04 & $1: 172$ \\
\hline 754.5 & Pie equino varo & 11 & 6,25 & 19,35 & $1: 517$ \\
\hline 754.6 & Pie calcáneo valgo & 1 & 0,57 & 1,76 & $1: 5.686$ \\
\hline 755.01 & Polidactilia preaxial en pies & 3 & 1,70 & 5,28 & $1: 1.895$ \\
\hline 755.03 & Polidactilia preaxial en manos & 1 & 0,57 & 1,76 & $1: 5.686$ \\
\hline 755.1 & Sindactilia ósea en manos & 1 & 0,57 & 1,76 & $1: 5.686$ \\
\hline 755.12 & Sindactilia ósea en pies & 1 & 0,57 & 1,76 & $1: 5.686$ \\
\hline 755.24 & Ausencia de manos o dedos & 3 & 1,70 & 5,28 & $1: 1.895$ \\
\hline 755.25 & Brazos cortos & 2 & 1,14 & 3,52 & $1: 2.843$ \\
\hline 755.33 & Ausencia de miembro inferior & 1 & 0,57 & 1,76 & $1: 5.686$ \\
\hline 755.80 & Artrogriposis múltiple congénita & 2 & 1,14 & 3,52 & $1: 2.843$ \\
\hline
\end{tabular}




$\begin{array}{ll}756.41 & \text { Condrodisplasia (acondrogénesis) } \\ 756.44 & \begin{array}{l}\text { Otros síndromes de enanismo especificados† } \\ \text { (displasia tanatofórica) }\end{array} \\ 756.5 & \text { Osteogénesis imperfecta } \\ 756.61 & \text { Hernia diafragmática congénita } \\ 756.7 & \text { Onfalocele } \\ 756.71 & \text { Gastrosquisis } \\ 756.72 & \text { Síndrome de prune belli† } \\ 758.0 & \text { Trisomía 21 } \\ 758.1 & \text { Trisomía 13 } \\ 758.2 & \text { Trisomía 18 } \\ 758.6 & \text { Monosomía X } \\ 778 & \text { Hidrops fetalis no inmune† } \\ \text { Total } & \end{array}$

$\begin{array}{rrrr}1 & 0,57 & 1,76 & 1: 5686 \\ 1 & 0,57 & 1,76 & 1: 5686 \\ 2 & 1,14 & 3,52 & 1: 2843 \\ 2 & 1,14 & 3,52 & 1: 2843 \\ 1 & 0,57 & 1,76 & 1: 5686 \\ 5 & 2,84 & 8,79 & 1: 1137 \\ 1 & 0,57 & 1,76 & 1: 5686 \\ 14 & 7,95 & 24,62 & 1: 406 \\ 1 & 0,57 & 1,76 & 1: 5686 \\ 2 & 1,14 & 3,52 & 1: 2843 \\ 1 & 0,57 & 1,76 & 1: 5686 \\ 4 & 2,27 & 7,03 & 1: 1422 \\ 169 & 100 & & \end{array}$

Cuadro 2. Anomalías congénitas moderadas clasificadas de acuerdo con el Código Internacional de las Enfermedades 9 (CIE-9).

\begin{tabular}{|c|c|c|c|c|c|}
\hline \multirow[t]{2}{*}{ CIE-9 } & \multirow[t]{2}{*}{ Descripción de la malformación } & \multicolumn{2}{|c|}{ Frecuencia } & \multirow{2}{*}{$\begin{array}{c}\text { Tasa por } \\
10.000\end{array}$} & \multirow{2}{*}{$\begin{array}{c}\text { Nacimientos } \\
\text { por caso }\end{array}$} \\
\hline & & $\mathbf{n}$ & $\%$ & & \\
\hline 228.01 & Hemangioma de la piel & 4 & 5,26 & 7,03 & $1: 1.422$ \\
\hline 228.1 & Linfangioma & 1 & 1,32 & 1,76 & $1: 5.686$ \\
\hline 528.4 & Quiste oral & 1 & 1,32 & 1,76 & $1: 5.686$ \\
\hline 685.1 & Seno coccígeo & 11 & 14,47 & 19,35 & $1: 517$ \\
\hline 743.34 & Coloboma del iris & 1 & 1,32 & 1,76 & $1: 5.686$ \\
\hline 744.12 & Apéndice preauricular & 21 & 27,63 & 36,93 & $1: 271$ \\
\hline 744.41 & Seno prearuricular & 9 & 11,84 & 15,83 & $1: 632$ \\
\hline 750.18 & Otras anomalías, especialmente de lengua (frenillo breve) & 2 & 2,63 & 3,52 & $1: 2.843$ \\
\hline 752.4 & Anomalías genitales externos femeninos (clitoromegalia) & 2 & 2,63 & 3,52 & $1: 2.843$ \\
\hline 752.6 & Hipospadia (grado I) & 2 & 2,63 & 3,52 & $1: 2.843$ \\
\hline 755.00 & Polidactilia postaxial en manos & 8 & 10,53 & 14,07 & $1: 711$ \\
\hline 755.02 & Polidactilia postaxial en pies & 3 & 3,95 & 5,28 & $1: 1.895$ \\
\hline 755.11 & Sindactilia cutánea en manos & 1 & 1,32 & 1,76 & $1: 5.686$ \\
\hline 755.13 & Sindactilia cutánea en pies & 1 & 1,32 & 1,76 & $1: 5.686$ \\
\hline 755.5 & Anomalías de los dedos (camptodactilia) & 1 & 1,32 & 1,76 & $1: 5.686$ \\
\hline 757.38 & Nevo no clasificable & 2 & 2,63 & 3,52 & $1: 2.843$ \\
\hline 757.4 & Alopecia congénita & 3 & 3,95 & 5,28 & $1: 1.895$ \\
\hline 757.62 & Mamila supernumeraria & 3 & 3,95 & 5,28 & $1: 1.895$ \\
\hline Total & & 76 & 100 & & \\
\hline
\end{tabular}

frecuencia de anomalías $(4,16 \%$ y $4,76 \%$, $p=0,2986$ ). En un estudio previo realizado en el IMI en 1993 (30), se registró una frecuencia de $2,68 \%$ de niños con anomalías congénitas en 9.224 nacimientos vivos; sin embargo, esta cifra no incluye el registro de anomalías moderadas. Al comparar sólo las anomalías mayores de nuestro estudio con el estudio previo ( $3 \%$ vs. $2,6 \%$ ), se encuentra que no difieren estadísticamente $(p=0,1840)$, lo que indica que en el periodo transcurrido no ha habido una variación en las frecuencias a pesar de que el IMI ha mostrado cambios importantes en el número de nacimientos en las épocas mencionadas. Estas cifras son semejantes a las informadas en la literatura internacional (33) y en algunos estudios nacionales (34-37) (cuadro 4).

Las diferencias observadas entre el presente estudio y los de Isaza (35) y Pinto (36) podrían explicarse por los criterios de inclusión, lo que conlleva subregistro de algunas anomalías.

Por otra parte, diferentes estudios internacionales realizados en las décadas de 1960 (38) y de 1970 (28) muestran cifras muy altas de frecuencias de anomalías congénitas: 15,84\% en el primero, por 
Cuadro 3. Distribución y análisis de las variables del estudio, discriminadas por anomalías mayores, moderadas y totales.

\begin{tabular}{|c|c|c|c|c|c|c|c|c|c|}
\hline \multirow{3}{*}{$\begin{array}{l}\text { Variable } \\
\text { analizada }\end{array}$} & \multicolumn{3}{|c|}{ Anomalías mayores } & \multicolumn{3}{|c|}{ Anomalías moderadas } & \multicolumn{3}{|c|}{ Todas las anomalías } \\
\hline & $\begin{array}{l}\text { Casos } \\
n=169\end{array}$ & $\begin{array}{r}\text { Control } \\
n=169\end{array}$ & & $\begin{array}{c}\text { Casos } \\
n=76\end{array}$ & $\begin{array}{c}\text { Controles } \\
n=76\end{array}$ & & $\begin{array}{l}\text { Casos } \\
n=245\end{array}$ & $\begin{array}{r}\text { Contro } \\
\mathrm{n}=245\end{array}$ & $\begin{array}{l}\text { les } \\
5\end{array}$ \\
\hline & $\mathbf{x}$ & $\mathbf{x}$ & p† & $\mathbf{x}$ & $\mathbf{x}$ & pt & $\mathbf{x}$ & $\mathbf{x}$ & pt \\
\hline Edad materna & 25,51 & 25,35 & 0,8405 & 24,98 & 26,09 & 0,3455 & 25,35 & 25,57 & 0,7317 \\
\hline Edad paterna & 28,76 & 28,31 & 0,6438 & 29,38 & 28,054 & 0,3835 & 28,95 & 28,21 & 0,3667 \\
\hline Edad de gestación & 36,17 & 36,84 & 0,0717 & 37,48 & 37,13 & 0,48 & 36,57 & 36,9 & 0,2161 \\
\hline Peso recién nacido & lo 2640,92 & 2848,81 & 0,0033 & 2982,19 & 2943,06 & 0,6502 & 2746,78 & 2875,33 & 0,0228 \\
\hline
\end{tabular}

\begin{tabular}{|c|c|c|c|c|c|c|c|c|c|c|c|c|c|c|c|}
\hline & $\mathbf{n}$ & $\%$ & $\mathbf{n}$ & $\%$ & $\mathrm{p} \dagger$ & $\mathbf{n}$ & $\%$ & $\mathbf{n}$ & $\%$ & $\mathrm{p} \dagger$ & $\mathbf{N}$ & $\%$ & $\mathbf{n}$ & $\%$ & p† \\
\hline peso $<2.500 \mathrm{~g}$ & 67 & 39,6 & 33 & 19,53 & 0,000 & 13 & 17,1 & 12 & 16,0 & 0,855 & 80 & 32,6 & 45 & 18,3 & 0,000 \\
\hline peso $<1.500 \mathrm{~g}$ & 9 & 5,33 & 9 & 5,33 & 1 & 1 & 1,3 & 2 & 2,7 & 0,552 & 10 & 4,0 & 11 & 4,5 & 0,823 \\
\hline Alta muerto & 41 & 24,2 & 4 & $2,3 \%$ & 0,000 & 1 & 1,3 & 2 & 2,7 & 0,552 & 42 & 17,1 & 6 & 2,5 & 0,000 \\
\hline Antec. de aborto & 26 & 15,38 & 24 & 14,04 & 0,725 & 12 & 15,8 & 12 & 16,0 & 0,972 & 38 & 15,1 & 35 & 14,3 & 0,703 \\
\hline $\begin{array}{l}\text { Antecedentes de } \\
\text { consanguinidad }\end{array}$ & 8 & 4,73 & 5 & 2,92 & 0,384 & 1 & 1,3 & 0 & 0,0 & 0,319 & 9 & 3,7 & 5 & 2,0 & 0,278 \\
\hline $\begin{array}{l}\text { Antec. de } \\
\text { anomalías } \\
\text { familiares }\end{array}$ & 44 & 26,0 & 14 & 8,2 & 0,000 & 31 & 40,8 & 11 & 14,7 & 0,000 & 75 & 30,6 & 25 & 10,2 & 0,000 \\
\hline $\begin{array}{l}\text { Multivitamínicos } \\
\text { periconcepcionales }\end{array}$ & 17 & 10,03 & 23 & 13,45 & 0,332 & 9 & 11,8 & 15 & 20,0 & 0,17 & 26 & 10,6 & 38 & 15,5 & 0,108 \\
\hline Enf. agudas* & 64 & 37,8 & 56 & 32,75 & 0,323 & 34 & 44,7 & 26 & 34,7 & 0,206 & 98 & 40,0 & 81 & 33,2 & 0,111 \\
\hline Inmunizaciones* & 40 & 23,6 & 45 & 26,3 & 0,573 & 26 & 34,2 & 18 & 24,0 & 0,167 & 66 & 26,9 & 63 & 25,7 & 0,758 \\
\hline Enf. crónicas* & 20 & 11,8 & 19 & 11,1 & 0,834 & 11 & 14,5 & 13 & 17,3 & 0,631 & 31 & 12,6 & 32 & 13,1 & 0,893 \\
\hline Fact. físicos* & 13 & 7,6 & 8 & 4,7 & 0,248 & 8 & 10,5 & 5 & 6,7 & 0,398 & 21 & 8,6 & 13 & 5,3 & 0,155 \\
\hline Medicamento* & 78 & 46,2 & 85 & 49,7 & 0,512 & 36 & 47,4 & 32 & 42,7 & 0,561 & 114 & 46,51 & 116 & 47,3 & 0,856 \\
\hline Alcohol* $^{*}$ & 5 & 3,0 & 7 & 4,1 & 0,571 & 7 & 9,2 & 4 & 5,3 & 0,359 & 12 & 4,9 & 11 & 4,5 & 0,831 \\
\hline Cigarrillo* & 10 & 5,9 & 13 & 7,6 & 0,536 & 6 & 7,9 & 8 & 10,7 & 0,557 & 16 & 6,5 & 21 & 8,6 & 0,393 \\
\hline Drogas* & 2 & 1,2 & 0 & 0,0 & 0,154 & 0 & 0 & 0 & 0 & - & 2 & 0,8 & 0 & 0,0 & 0,153 \\
\hline Plaguicidas* & 5 & 2,9 & 4 & 2,3 & 0,722 & 0 & 0,0 & 4 & 5,3 & 0,041 & 5 & 2,0 & 8 & 3,3 & 0,399 \\
\hline Metrorragia* & 22 & 13,02 & 18 & 10,5 & 0,476 & 3 & 3,9 & 10 & 13,3 & 0,04 & 25 & 10,2 & 28 & 11,4 & 0,663 \\
\hline
\end{tabular}

* Primer trimestre del embarazo; $† \alpha=0,05$

Cuadro 4. Comparación de las frecuencias observadas en algunos estudios realizados en el país con las del presente estudio.

\begin{tabular}{lccc}
\hline Autor, año (ref.) & $\mathbf{n}$ & $\%$ & $\boldsymbol{p}$ \\
\hline Silva, 1984 (34) & 6.805 & 3,20 & 0,6809 \\
Isaza, 1989 (35) & 9.103 & 2,34 & 0,0076 \\
Pinto,1990 (36) & 8.469 & 2,18 & 0,0012 \\
Giraldo,1992 (37) & 7.752 & 2,73 & 0,2600 \\
Arteaga, 1993 (30) & 9.224 & 2,68 & 0,1840 \\
Presente estudio, 2000 & 5.686 & $3,0^{*}$ & - \\
\hline
\end{tabular}

* Sólo anomalías mayores

incluir un número importante de niños con una sola anomalía menor, y $15,5 \%$ en el segundo, por incluir, igualmente, aunque en menor proporción, niños con una sola anomalía menor y, además, registrar todo tipo de anomalías durante los primeros siete años de vida. Como se ha mencionado anteriormente, una anomalía menor aislada en niños por lo demás normales, se considera una variante normal del desarrollo.

Llama la atención que la mayor frecuencia en las anomalías mayores se observó en la displasia congénita de la cadera $(18,75 \%, 1: 170)$, el síndrome de Down $(7,95 \%, 1: 400)$, el pie equino $(6,35 \%, 1: 516)$, la microtia $(3,4 \%, 1: 950)$ y la criptorquidia bilateral $(3,4 \%, 1: 950)$. Se notó un aumento de casos con relación a los esperados según datos del ECLAMC 1982-1998 para la displasia congénita de la cadera $(a<0,01)$; las otras cifras no son significativamente altas al compararlas con las del ECLAMC (cuadro 5).

La mayor frecuencia de la displasia congénita de cadera se explica por un alto interés en esta anomalía durante la evaluación del neonato, lo que posiblemente ocasiona un exceso de registro; es interesante anotar que frecuentemente se 
Cuadro 5. Frecuencias encontradas en el presente estudio, comparadas con un estudio previo del IMI (Arteaga, 1993) con base en los hospitales de Suramérica, ECLAMC (1982-1998).

\begin{tabular}{|c|c|c|c|c|c|c|c|c|c|c|c|}
\hline \multirow[b]{2}{*}{ ICD 9} & \multirow{2}{*}{ Descripción de la malformación } & \multicolumn{2}{|c|}{$\begin{array}{c}\text { ECLAMC } \\
\text { (1982-1998) } \\
\text { Total nacimientos: } \\
\text { 3'020.896 }\end{array}$} & \multicolumn{4}{|c|}{$\begin{array}{c}\text { IMI } \\
\text { (Arteaga, 1991-1992) } \\
\text { Total nacimientos: } \\
9.224\end{array}$} & \multicolumn{4}{|c|}{$\begin{array}{l}\text { I.M.I. } \\
\text { esente estudio 1997-2000) } \\
\text { Total nacimientos: } \\
5.686\end{array}$} \\
\hline & & & $\begin{array}{c}\mathrm{T} \\
\times 10.000\end{array}$ & & $\begin{array}{c}\mathrm{T} \\
\mathrm{n} \times 10.000\end{array}$ & $0 \quad \lambda^{*}$ & $\mathrm{IC} \dagger$ & & $\begin{array}{c}\mathrm{T} \\
\mathrm{n} \times 10.000\end{array}$ & $\lambda^{*}$ & $\mathrm{IC} \dagger$ \\
\hline 740.02 & Anencefalia & 2087 & 6,91 & 11 & 11.93 & 6.37 & $2-16$ & 4 & 7,03 & 3,9 & $1-13$ \\
\hline 741 & Espina bífida & 2299 & 7,61 & 5 & 5.42 & 7.02 & $2-17$ & 9 & 15,83 & 4,3 & $1-13$ \\
\hline 742.00 & Encefalocele occipital & 594 & 1,97 & 1 & 1.08 & 1.81 & $0-9$ & 2 & 3,52 & 1,1 & $0-7$ \\
\hline 742.1 & Microcefalia & 840 & 2,78 & 0 & 0.00 & 2.56 & $0-11$ & 2 & 3,52 & 1,6 & $0-9$ \\
\hline 742.3 & Holoprosencefalia & - & - & 5 & 5.42 & - & - & 3 & 5,28 & - & - \\
\hline 742.3 & Hidrocefalia & 2224 & 7,36 & 14 & 15.18 & 6.79 & $2-17$ & 8 & 14,07 & 4,2 & $1-13$ \\
\hline 742.4 & Macrocefalia & - & - & 1 & 1.08 & - & - & 1 & 1,76 & - & - \\
\hline 744.2 & Microtia & 1267 & 4,19 & 3 & 3.25 & 3.87 & $1-13$ & 6 & 10,55 & 2,4 & $0-9$ \\
\hline 745.4 & Defecto septal ventricular & 2438 & 8,07 & 9 & 9.76 & 7.44 & $2-17$ & 3 & 5,28 & 4,6 & $1-14$ \\
\hline 746.1 & Atresia tricuspídea congénita & - & - & 1 & 1.08 & - & - & 1 & 1,76 & - & - \\
\hline 746.7 & $\begin{array}{l}\text { Síndrome de corazón izquierdo } \\
\text { hipoplásico }\end{array}$ & - & - & 2 & 2.17 & - & - & 1 & 1,76 & - & - \\
\hline 749.2 & Labio y paladar hendido & 3291 & 10,89 & 9 & 9.76 & 10.05 & $4-21$ & 4 & 7,03 & 6,2 & $2-16$ \\
\hline 750.3 & Estenosis esofágica & 852 & 2,82 & 1 & 1.08 & 2.60 & $0-11$ & 6 & 10,55 & 1,6 & $0-9$ \\
\hline 751.1 & Estenosis del duodeno & 274 & 0,91 & 0 & 0.00 & 0.84 & $0-7$ & 1 & 1,76 & 0,5 & $0-7$ \\
\hline 751.1 & Estenosis de yeyuno e ileon & 197 & 0,65 & 0 & 0.00 & 0.60 & $0-7$ & 2 & 3,52 & 0,4 & $0-5$ \\
\hline 751.5 & Fístula anal congénita & - & - & 1 & 1.08 & - & - & 1 & 1,76 & - & - \\
\hline 752.5 & $\begin{array}{l}\text { Testículos no descendidos } \\
\text { bilaterales }\end{array}$ & - & - & 3 & 3.25 & - & - & 6 & 10,55 & - & - \\
\hline 752.6 & Hipospadias & 2499 & 8,27 & 1 & .08 & 7.63 & $3-199$ & 3 & 5,28 & 4,7 & $1-14$ \\
\hline 753.00 & Secuencia de Potter & - & - & 1 & .08 & - & - & 2 & 3,52 & - & - \\
\hline 753.01 & $\begin{array}{l}\text { Ausencia, agenesia o displasia } \\
\text { de riñón }\end{array}$ & 405 & 1,34 & 0 & 0.00 & 1.24 & $0-7$ & 1 & 1,76 & 0,8 & $0-7$ \\
\hline 753.10 & Riñón poliquístico & 616 & 2,04 & 6 & 6.50 & 1.88 & $0-9$ & 2 & 3,52 & 1,2 & $0-7$ \\
\hline 753.20 & Hidronefrosis congénita & 891 & 2,95 & 3 & 3.25 & 2.72 & $0-11$ & 2 & 3,52 & 1,7 & $0-9$ \\
\hline 756.7 & Secuencia de prune belli & - & - & 1 & 1.08 & - & - & 1 & 1,76 & - & - \\
\hline 754.30 & Displasia congénita de la cadera & 4785 & 15,8 & 12 & 13.01 & 14.61 & $7-28$ & 33 & 58,04 & 9,0 & 3-20‡ \\
\hline 754.50 & Pie equino varo & 5077 & 16,81 & 7 & 7.59 & 15.50 & $8-299$ & 11 & 19,35 & 9,6 & $4-21$ \\
\hline 754.60 & neo valgo & 2021 & 6,69 & 0 & 0.00 & 6.17 & $2-16 \rrbracket$ & 1 & 1,76 & 3,8 & $1-13$ \\
\hline 755.0 & preaxial & 780 & 2,5 & - & - & - & - & 4 & 7,03 & 1,5 & $0-9$ \\
\hline 755.0 & Polid & 4805 & 15,91 & - & - & - & - & 11 & 19,35 & 9,0 & $3-20$ \\
\hline 755.0 & (ambos tinos) & - & - & 5 & 5.42 & - & - & 15 & 26,38 & - & - \\
\hline 755.2 & Ausencia de manos o dedos & - & - & - & - & - & - & 3 & 5,28 & - & - \\
\hline 755.3 & Defecto de reducción de miembros & - & - & - & - & - & - & 6 & 10,55 & - & - \\
\hline 755.5 & Anomalías de los dedos & - & - & 1 & 1.08 & - & - & 1 & 1,76 & - & - \\
\hline 755.8 & Artrogriposis múltiple congénita & 480 & 1,59 & 0 & 0.00 & 1.47 & $0-7$ & 2 & 3,52 & 0,9 & $0-7$ \\
\hline 756.41 & Condrodisplasia & - & - & 1 & 1.08 & - & - & 1 & 1,76 & - & - \\
\hline 756.44 & Otros síndromes de enanismo esp. & - & - & 1 & 1.08 & - & - & 1 & 1,76 & - & - \\
\hline 756.5 & Osteogénesis imperfecta & - & - & 1 & 1.08 & - & - & 2 & 3,52 & - & - \\
\hline 756.6 & Hernia diafrágmatica congénita & 644 & 2,13 & 2 & 2.17 & 1.97 & $0-9$ & 2 & 3,52 & 1,2 & $0-7$ \\
\hline 756.7 & Onfalocele & 742 & 2,46 & 1 & 1.08 & 2.27 & $0-9$ & 1 & 1,76 & 1,4 & $0-7$ \\
\hline 756.7 & Gastrosquisis & 345 & 1,14 & 1 & 1.08 & 1.05 & $0-7$ & 5 & 8,79 & 0,6 & $0-7$ \\
\hline 757.4 & Nevo no clasificable & - & - & 1 & 1.08 & - & - & 2 & 3,52 & - & - \\
\hline 758.0 & Trisomía 21 & 4778 & 15,82 & 13 & 14.01 & 14.59 & $7-28$ & 14 & 24,62 & 9,0 & $3-20$ \\
\hline
\end{tabular}

*Valor esperado para esta serie; † intervalo de confianza para distribución de Poisson con $\alpha=0,01$; $\ddagger$ aumento estadísticamente significativo; I disminución estadísticamente significativa 
confunden los click de inmadurez de la cadera con signos de luxación (Barlow y Ortolani), por eso es necesario confirmarlos con el ortopedista o por ecografía de caderas; sin embargo, estos recursos no estaban disponibles durante la realización del estudio. En la experiencia del equipo es de resaltar la importancia, en orden decreciente, de los siguientes signos clínicos: Ortolani, Barlow, asimetría de miembros, limitación en la abducción de las caderas y asimetría de pliegues en cara posterior del muslo. Al comparar los datos del estudio precedente en el IMI, 1991-92, se registraron menos casos de lo esperado para hipospadias, pie equino, pie calcáneo valgo y polidactilia postaxial, lo cual podría deberse a un subregistro.

En el presente estudio, las anomalías moderadas más frecuentes fueron los apéndices preauriculares $(27,63 \%, 1: 270)$; los senos coccígeos $(14,47 \%, 1: 516)$; las polidactilias postaxiales (14,47\%; 1:710); la fístula preauricular $(11,84 \%, 1: 631)$ y los hemangiomas $(5,26 \%$, $1: 142)$. Es importante resaltar el papel del pediatra en el diagnóstico temprano y en el manejo de las anomalías moderadas y la búsqueda de posibles anomalías asociadas en otros órganos tales como asociaciones renales y pérdida de la audición en pacientes con fístulas y apéndices preauriculares $(39,40)$; espina bífida oculta en pacientes con senos dérmicos sacros (41); alteraciones renales asociadas con pezones supernumerarios $(42,43)$ y manchas café con leche asociadas con neurofibromatosis (44). Así mismo, se resalta la importancia de reconocer tres o más anomalías menores como posibles predictores de anomalías mayores $(38,45)$.

En este estudio, el análisis estadístico entre casos y controles reflejó una mayor mortalidad en los niños con anomalías congénitas al ser dados de alta, así como también su bajo peso al nacer. El antecedente familiar de anomalías congénitas en parientes de primer grado también resultó significativo, lo cual está acorde con lo informado por Giraldo (37), e indica una importante asociación con el riesgo de presentar anomalías congénitas. Es importante señalar que el único factor que estuvo asociado con las anomalías moderadas es el antecedente familiar de anomalías congénitas. Esto último confirma el carácter genético (monogénico o multifactorial) de dichas anomalías moderadas.

Nuestra observación acerca de la posible etiología de las anomalías congénitas está de acuerdo con lo informado en la literatura, en la cual se señala que las causas mendelianas están entre $3 \%$ y $8 \%$, las cromosómicas entre $6 \%$ y $13 \%$, las multifactoriales entre $20 \%$ y $51 \%$ y las de etiología desconocida entre $29 \%$ y $61 \%$ (33).

En conclusión, la vigilancia epidemiológica tiene como finalidad la prevención y control de los problemas de salud. Su aplicación requiere de un trabajo multidisciplinario activo y permanente $y$, debido a que está estructurada como un proceso dinámico y continuo de evaluación y control, interrelaciona permanentemente la observación, el análisis y la intervención.

El desafío de los países en desarrollo respecto al estudio y manejo de las anomalías congénitas debe centrarse en la implementación de un sistema continuo y confiable de base hospitalaria, como el ECLAMC/VERACC, o poblacional, como el de los países europeos, si no es posible de todas las anomalías, por lo menos de algunas 'anomalías centinela' (síndrome de Down, labio y paladar hendido, defectos del tubo neural, hiperplasia suprarrenal, por citar sólo algunas), que permita conocer los datos demográficos básicos, establecer la prevalencia de las anomalías, detectar la prevalencia de factores de riesgo, como fue descrito recientemente por Muñoz (46), e implementar programas de intervención, como se ha intentado en Neiva (Colombia) con la suplementación de ácido fólico (47).

El presente estudio constituye un esfuerzo por agrupar las anomalías congénitas mayores y moderadas, clasificarlas sistemáticamente de acuerdo con CIE-9, compararlas con referentes internacionales y estimar su frecuencia. Además, pretende aportar elementos en la implementación de programas de vigilancia de anomalías congénitas en el país.

\section{Agradecimientos}

Este estudio fue financiado parcialmente por Colciencias a través de su programa Jóvenes 
Investigadores, Convenio 007.2001. Se agradece a los médicos Harvy Velazco, Abel Bermeo y Edgar Peñaranda por su colaboración en la recolección de datos y a todos los médicos pediatras y ginecoobstetras, al igual que al personal de enfermería del Instituto Materno Infantil por su contribución en la realización del presente estudio.

\section{Referencias}

1. Medina MR, Martínez C, Gutiérrez JA. Geografía de la mortalidad infantil en Colombia, 1985-1994. Bogotá: Imprenta Dane; 1999.

2. Profamilia. Encuesta Nacional de Demografía y Salud 2000. Bogotá: Profamilia, Printex Impresores Ltda; 2000.

3. World Health Organization. Causes of infant death, by sex and age. Wld Hith Statist Annu 1987:414-42.

4. Sever LE, Lynberg MC, Edmonds LD. The impact of congenital malformation on public health. Teratology 1993;48:547-9.

5. Cunniff C, Carmack JL, Kirby RS, Fiser DH. Contribution of heritable disorders to mortality in the pediatric intensive care unit. Pediatrics 1995;95:67881.

6. Kempe A, Wise DH, Wampler NS, Cole FS, Wallace H, Dickson C, et al. Risk status at discharge and cause of death for postneonatal infant deaths: a total population study. Pediatrics 1997;99:338-44.

7. Rice DP, Hodgson TA, Kopstein AN. The economic cost of illness; a replication and update. Health Care Finan Rev 1985;7:61-80.

8. Ministerio de Salud. La carga de la enfermedad en Colombia. Bogotá: Editorial Carrera Séptima; 1994.

9. Gallardo HM, Rodríguez J. La carga de la enfermedad en Santafé de Bogotá. Bogotá: Panamericana Formas e Impresos; 1999.

10. Stoll C, Roth MP, Dott B, Bigel P. Usefulness of a registry of congenital malformations for genetic counseling and prenatal diagnosis. Clin Genet 1986; 29:204-10.

11. Källén B, Winberg JA. Swedish register of congenital malformations. Experience with continuous registration during two years with special reference to multiple malformations. Pediatrics 1968;41:765-76.

12. Saxén L, Klemetti A, Härö AS. A matched-pair register for studies of selected congenital defects. Am J Epidemiol 1974;100:297-306.

13. Kucera J. Congenital malformations surveillance systems. Int J Epidemiol 1986;15:430.

14. International Clearinghouse for Birth Defects Monitoring Systems. A communication from the
International Clearinghouse for Birth Defects Monitoring Systems. Int J Epidemiol 1981;10:245-6.

15. Robert M, Robert E, Francannet C. The International Clearinghouse for Birth Defects Information Systems (ICBDIS). Un exemple de coopération international efficace. Arch Fr Pediatr 1986;43:539-42.

16. Lechat MF, De Walls P, Weatherall J. European Economic Community's concerted action on congenital anomalies: The Eurocat project. Prog Clin Biol Res 1985; 163B:11-15.

17. De Wals $\mathbf{P}$, Dolk $\mathbf{H}$, Bertrand $\mathbf{F}$, Gillerot $\mathbf{Y}$, Weatherall $\mathbf{J}$, Lechat $\mathbf{M}$. La surveillance épidémiologique des anomalies congénitales par le registre EUROCAT. Rev Epidém et Santé Publ 1988;36:273-82.

18. Källén B, Bertollini R, Castilla E, Czeizel A, Knudsen LB, Martínez-Frías ML, et al. A joint international study on the epidemiology of hypospadias. Acta Paediatr Scand 1986;324:1-52.

19. Castilla EE, Orioli IM. Estudio colaborativo latinoamericano de anomalías congénitas: ECLAMC/ MONITOR. Interciencia 1983;3:271-8.

20. López-Camelo SJ, Orioli IM. Heterogeneus rates for birth defects in Latin America: hints of causality. Genet Epidemiol 1996;13:469-81.

21. Penchaszadeh V, Beiguelman. Medical genetics services in Latin America: report of a meeting of experts. Pan Am J Public Health 1998;3:409-20.

22. The British Pediatric Association. British Paediatric Association classification of diseases. London: The Office of Population Censusses and Surveys; 1979.

23. The Congenital Malformation Registry of New York. Reportable ICD codes, 1998. Disponible en: URL: http://www.health.state.ny.us/nysdoh/cmr/cmricd.htm.

24. Spranger J, Benirschke K, Hall JG, Lenz W, Lowry RB, Opitz JM, et al. Errors of morphogenesis: concepts and terms. Recommendations of an international working group. J Pediat 1982;100:160-5.

25. Opitz JM. What the general pediatrician should know about developmental anomalies. Pediat Rev 1982;3:26771.

26. Opitz JM. Study of minor anomalies in childhood malignancy. Eur J Pediatr 1985;144:252-4.

27. Opitz JM, Czeizel A, Evans JA, Hall JG, Lubinsky MS, Spranger JW. Nosologic grouping in birth defects. Editores: Vogel S, Sperling K. Berlin: 1987; p. 383-5.

28. Chung CS, Myrianthopoulos NC. Congenital anomalies: mortality and morbidity, burden and classification. Am J Med Genet 1987;27:505-23.

29. Mckusick VM. Mendelian inheritance in man. Eleventh edition. Baltimore: Johns Hopkins University Press; 1994. 
30. Arteaga CE. Prevalencia y factores asociados a las anomalías congénitas y enfermedades de instalación prenatal en el Instituto Materno Infantil de Bogotá (tesis). Bogotá: Universidad Nacional de Colombia; 1993.

31. Estudio Colaborativo Latinoamericano de Malformaciones Congénitas. XXI Reunion anual del Estudio Colaborativo Latinoamericano de Malformaciones Congénitas, 2000. Disponible en: URL: http://www.biologia.ufrj.br/sociedades/eclamc.

32. Robe-Hesketh S, Everi HB. A handbook of statistical analysies using Stata. Second edition. Texas: Chapman \& Holl/CRC; 2000

33. Kalter $\mathbf{H}$, Warkany J. Congenital malformation. Etiologic factors and their role in prevention. New Eng $\mathrm{J}$ Med 1983;308:424-31.

34. Silva JR. Evaluación genética y estudio de malformaciones congénitas. Acta Pediatrica Colombiana 1984;2:12-23.

35. Isaza C, Martina D, Estupiñán J, Stark C, Rey H. Prevalencia de anomalías congénitas diagnosticadas en las primeras 24 horas de vida. Colombia Médica 1989;20:156-9.

36. Pinto $\mathbf{P}$. Estudio de anomalías congénitas en una población de nacidos en el Instituto de Seguros Sociales (ISS) de Barranquilla, de 1985-1988. Salud Uninorte 1990;3:123-30.

37. Giraldo A. A case control study of clinically detected congenital anomalies in newborn babies and common risk factors (thesis). Baltimore: Johns Hopkins University; 1992.

38. Marden PM, Smith DW, McDonald MJ. Congenital anomalies in the newborn infant, including minor variation. J Pediatr 1964;64:357-71.
39. Kohelet D, Arbel E. A prospective search for urinary tract abnormalities in infant with isolated preauricular tag. Pediatrics 2000;105:E61.

40. Kugelman A, Hadad B, Ben-David J, Podoshin L, Borochowitz Z, Barder D. Preauricular yags and pits in the newborn: the role of hearing test. Acta Paediatr 1997;86:170-2.

41. Weprin BE, Oakes WJ. Coccygeal pits. Pediatrics 2000;105:E69.

42. Hersh JH, Bloom AS, Cromer AO, Harrison HL, Weisskopf B. Does a supernumerary nipple/renal field defect exist? Am J Dis Child 1987;141:989-91.

43. Kenney RD, Flippo JL, Black EB. Supernumerary nipples and renal anomalies in neonates. Am J Dis Child 1987;141:987-8.

44. Alper J, Holmes LB, Mihm MC. Birthmarks with serius significance: nevocellular nevi, sebaceus nevi, and multiple café au lait spots. J Pediatrics 1979;95:696700.

45. Lepping KA, Werler MM, Cann CL, Cook CA, Holmes LB. Predective value of minor anomalies. Asociation with major malformations. J Pediatr 1987; 110:530-7.

46. Muñoz J, Bustos I, Quintero Q, Giraldo A. Factores de riesgo para algunas anomalías congénitas en población colombiana. Rev Salud Pública 2001;3:26882.

47. Ostos H, Astaíza G, García F, Bautista M, Rojas F, Bermúdez A. Disminución de la incidencia de defectos del tubo neural en el Hospital Universitario de Neiva: posible efecto de la promoción del consumo de ácido fólico. Biomédica 2000;20:18-24. 